\title{
P57 In Patients with Chronic Descending Aorta Dissection the Maximum Systolic Flow Deceleration Rate in the False Lumen is Associated with Aortic Dilatation: a 4D-flow MRI Study
}

\author{
Andrea Guala ${ }^{1, *}$, Aroa Ruiz-Muñoz ${ }^{1}$, Lydia Duz-Santoy ${ }^{1}$, Gisela Teixido-Tura ${ }^{2}$, Chiara Granato ${ }^{2}$, Augusto Sao-Aviles ${ }^{1}$,
} Lucia La Mura ${ }^{2}$, Angela Lopez-Sainz ${ }^{2}$, Maria Luz Servato ${ }^{2}$, Arturo Evangelista ${ }^{1}$, Ignacio Ferreira ${ }^{2}$, Jose Rodriguez-Palomares ${ }^{2}$

${ }^{1}$ Vall d'Hebron Institute of Research, Barcelona, Spain

${ }^{2}$ Department of Cardiology, Vall d'hebron Hospital, Barcelona, Spain

\section{ABSTRACT}

Background: Aortic type B dissection is a life-threating condition in which blood flows between aortic wall layers. Despite a role for morphological variables, such as entry tear location and dimension, in the prediction of progressive false lumen (FL) dilation has been suggested, the role of flow characteristics has not been studied due to the unavailability of proper flow descriptions. Now that $4 \mathrm{D}$ phase-contrast magnetic resonance imaging (4D-flow MRI) allows for the evaluation of flow field with unprecedented detail, we tested whether flow deceleration in the FL, being a marker of occluded flow exit, is related to FL dilation.

Methods: In 29 patients with a patent FL and with a retrospective CT-based follow-up $>3$ years underwent 4D-flow MRI Flow acceleration in the FL was calculated in a standardized sub-volume of the descending aorta. The maximum systolic flow deceleration rate (MSDR) was quantified as maximum minus minimum acceleration during systole divided by the corresponding time interval (Figure 1a).

Results: Demographic and clinical variables were similar among growth-rate (GR) tertiles. MSDR was statistically different in patients with a GR $<1 \mathrm{~mm} /$ year (group 1) compared to fast-dilating patients (groups 2, 3) (Figure 1c). MSDR showed a positive linear correlation with GR $(R=0.48 ; p=0.008)$ (Figure $1 b)$.

Conclusion: The intensity of flow deceleration in the FL of chronic type B dissection is related to its retrospective growth rate. If confirmed in prospective studies, this marker may be useful to discriminate aorta enlargement and identify high-risk patients.
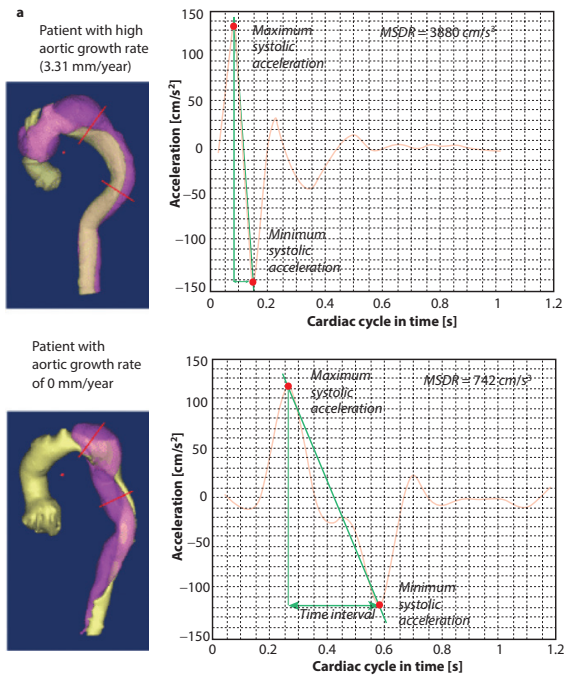
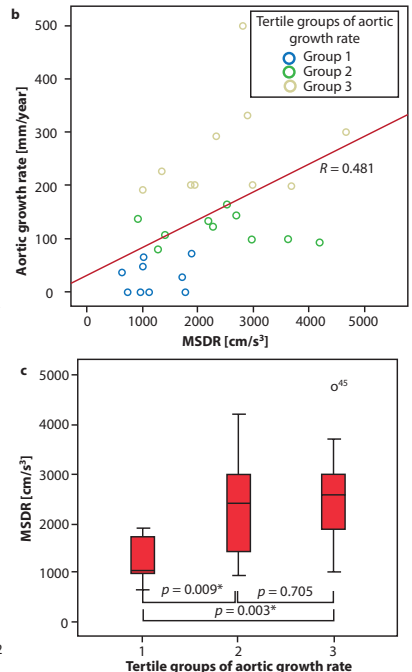

Figure 1 (a) Acceleration vs time curves extracted from the sub-volume of the false lumen (red) of two patients with aortic growth rates of 3.31 (top) and $0 \mathrm{~mm}$ /year (bottom). (b) Dispersion diagram of growth rate vs MSDR of flow in the false lumen. (c) Boxplot showing the trend of MSDR in the three tertiles of growth rate and the statistically significant difference between group 1 vs group 2 and 3 .

(c) 2019 Association for Research into Arterial Structure and Physiology. Publishing services by Atlantis Press International B.V. This is an open access article distributed under the CC BY-NC 4.0 license (http://creativecommons.org/licenses/by-nc/4.0/). 